\title{
THE FORMATION AND EVOLUTION OF GALAXIES:
}

\author{
Perspectives on the Origin of the Hubble Sequence
}

\author{
R.S. ELLIS \\ Institute of Astronomy \\ Cambridge, UK
}

\begin{abstract}
I review recent observational progress concerning the evolution of the morphological distribution of galaxies in the rich cluster environment and in the faint field population. By coupling HST imagery with ground-based spectroscopic diagnostics, evidence accumulates that galaxy morphology can be a transient phenomenon reflecting various changes in the star formation rate. Possible physical processes responsible for these changes are discussed. Future progress in understanding them will depend on securing 2-D spectroscopic data for representative systems. ${ }^{1}$
\end{abstract}

\section{Introduction}

It is a time of great progress in cosmology and galaxies remain the most useful fabric for studying the Universe, both at the present and early times. In this summary of a rapidly moving area, I have restricted myself to three broad results with two connecting themes. The first theme is concerned with the synergy achieved between space and ground-based facilities, particularly that between the refurbished Hubble Space Telescope (HST) and the new generation of 8-10 metre telescopes. The second more fundamental theme is related to the view that galaxy morphology is intimately connected with the nature and history of star formation. The growing body of HST data indicates that morphology may be a transient feature, reflecting short timescale changes in the star formation rate. Whilst this complicates traditional approaches towards galaxy evolution based on isolating population subsets according to morphological classifications, it provides an important insight into the physical processes governing star formation on galactic scales.

${ }^{1}$ To appear in IAU Symposium 183: Cosmological Parameters \& Evolution of the Universe, ed Sato, K. 
Thus far, the greatest progress has been made in studies based on properties integrated across the luminosity function i.e. for the detected population. In quantative detail, these results remains uncertain but new approaches based on the resolved properties of distant galaxies, taking advantage of both aspects stressed above, will allow us to study the physical processes responsible for the trends seen in the integrated populations and hence assist in explaining the distribution of morphological types seen at different epochs.

\section{The Role of the Environment}

One of the most fundamental observations relating to the origin of the Hubble sequence is the morphology-density relation (Dressler 1980). Does this dependence indicate that galaxy morphology was determined at birth with subsequent evolution occurring in a 'closed box' approximation? Or does it imply that galaxies have been transformed according to external processes? The first indications that the range of galaxy types is formed, at least for some systems, by nurture rather than nature, arose from the results of Butcher \& Oemler (1978). Using the fraction of luminous cluster members whose rest-frame colours were bluer than a fiducial value, those authors found the mean star formation rate in the cores of rich clusters was significantly higher 3-4 Gyr ago. HST images from a comprehensive survey of 10 clusters with $0.37<z<0.55$ undertaken by the 'Morphs' collaboration (Smail et al 1997a, Ellis et al 1997, Dressler et al 1997) have confirmed how this modest but important change in mean star formation rate in cluster environments is accompanied by a radical shift in the galaxy morphologies. The bulk of the blue light originally located by Butcher \& Oemler arises in disk galaxies (Figure 1) noticeably absent in present day cluster cores. Although Allington-Smith et al (1994) showed, on the basis of ground-based multi-colour photometry, that the rising blue fraction with redshift was a trend largely confined to rich clusters, the Morphs team has provided the first glimpse of the morphology-density relation at a redshift $\simeq 0.5$ using their extensive HST data.

Before discussing the morphological distributions quantitatively, one might question whether such classifications can be done reliably at such limits, even noting the superb performance of HST. Morphology is, by its nature, a somewhat subjective quantity (Naim et al 1995), but the availability of HST data has spurred much activity, both in reducing the subjective nature of galaxy classifications, in terms of indices based on image concentration and asymmetry (Abraham et al 1994), and in simulations which aim to quantify the effect of redshift on the visual appearance of galaxies of known morphological type (Abraham et al 1996a). These studies indicate 


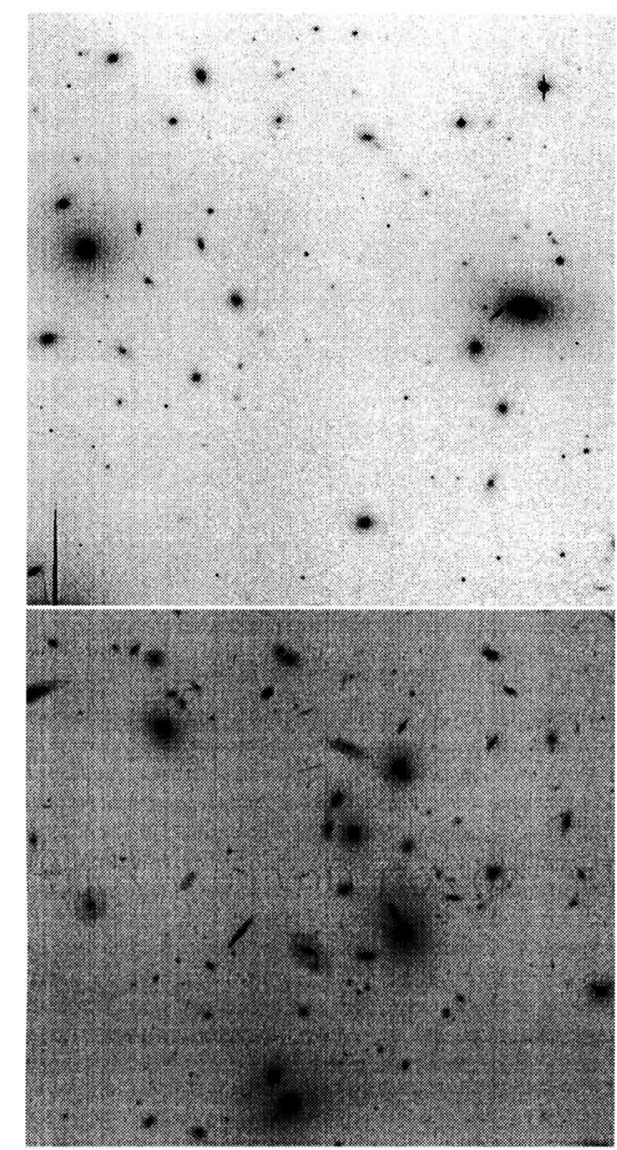

Figure 1. The transition in morphologies in the rich cluster environment since a redshift $z \simeq 0.3$. (Top) $R$ band ground-based image of the central $200 \mathrm{~h}^{-1} \mathrm{kpc}$ of the Coma cluster (courtesy of David Carter), (Bottom) HST F814W images of a comparable physical region in the distant cluster $A C 118$ ( $z=0.31)$

only modest shifts towards apparently later types at $z>0.7$ arising from surface brightness and bandshifting effects (Brinchmann et al 1997).

The Morphs data reveals a substantial change in the morphology-density relation at high redshift (Figure 2). The abundance of spirals is higher and less dependent on the projected surface density notwithstanding the reduced range at high $z$ c.f. locally (1.5 dex c.f. 2.5). Moreover, the abundance of S0s is 2-3 times less in proportion at high redshift. Whilst S0s could clearly be preferentially misclassified as Es, one would imagine this to be of greatest concern for face-on examples. In fact, the population ellipticity distribution shows no sign of a loss of face-on examples when compared to that determined locally (Dressler et al 1997). Figure 2 suggests that 

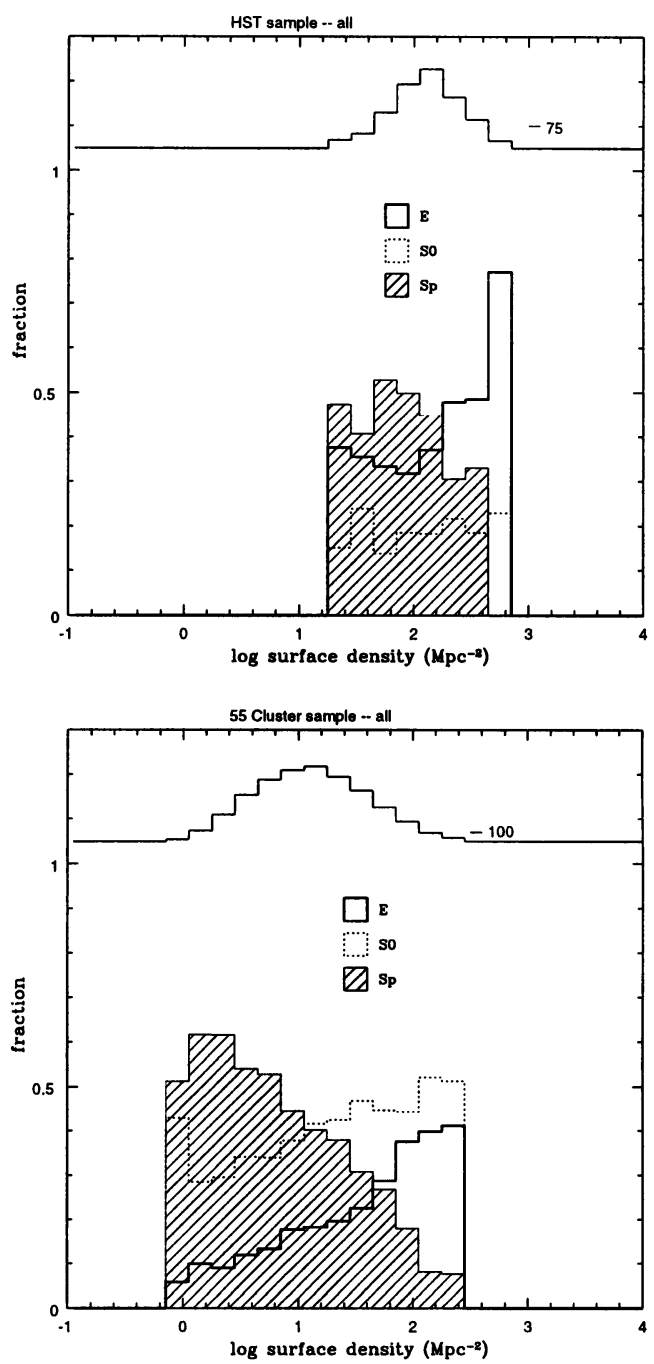

Figure 2. Evolution in the morphological fraction-projected surface density relation in the central $1.2 \mathrm{~h}^{-1} \mathrm{Mpc}$ regions of rich clusters from the analysis of Dressler et al (1997). (Top) 10 clusters with $0.37<z<0.57$ from the Morphs HST dataset, (Bottom) 55 local clusters reanalysed from the Dressler (1980) sample. The histogram in the top boxes show the number of galaxies in each bin.

present-day S0s must be counterparts of the intermediate star-forming disk galaxies and that the bulk of the Es are largely unaffected by whatever environmental processes occurred to the spirals.

Can the physical origin of the Butcher-Oemler effect really be so simple? Sandage \& Visvanathan (1978) and Bower et al (1992) demonstrated 
the significance of the small scatter in the rest-frame UV colour-magnitude relation. Using the Morphs data, Ellis et al (1997) find a remarkably small scatter $(<0.07 \mathrm{mag}$ in rest-frame U-V) in the colours of the elliptical population, even across different clusters, at $\mathrm{z} \simeq 0.5$ (see Stanford et al 1997 for an extension over a wider redshift range). The bulk of this population must be passively-evolving systems whose stars formed at an early time $(z>3)$ in broad agreement with earlier ground-based work (Aragon-Salamanca et al 1993). Similar constraints are now emerging from fundamental plane $(\mathrm{Mg}$ - $\sigma$ and $r_{e}-\sigma$ ) relations pursued to high redshift (van Dokkum \& Franx 1996, Bender et al 1997, Kelson et al 1997).

However, although some cluster ellipticals are undoubtedly old, it is by no means clear that all of the present day population formed in this way. Ellipticals could be continuously formed e.g. via mergers as clusters assemble, in a way that guarantees that at any epoch only the most quiescent, morphologically regular examples show minimal UV scatter. Only number density arguments can circumvent this bias and cluster samples are poorlysuited to such analyses. Indeed, Kauffmann (1995) has suggested that the richest systems selected at high $z$ are likely to be observed more immediately after their assembly than is the case locally and this would complicate any attempt to construct volume-limited samples for tracking the various populations.

Moreover, not all the present-day S0s can be transformed spirals either, as those few found at high redshift share the tight scatter of the ellipticals (Ellis et al 1997). And of course, red S0s are found in the local field which, presumably, cannot have been affected by those processes we claim are restricted to dense environments. To verify the simple picture above, one would ideally like to find examples of objects in transition. Smail et al (1997b) have penetrated further down the colour-luminosity relation in intermediate redshift clusters and found a marked increase in the UV scatter for otherwise passively-evolving systems. The dichotomy could be resolved following earlier conjectures that present-day S0s are of two types (van den Bergh 1990): luminous examples formed at birth and less luminous examples which may have formed from destroyed spirals. In this case, one would expect to locate strong luminosity trends in the recent evolution of disk galaxies. As may also be the case for the ellipticals, distinctive though a Hubble class may appear to be, not only may it be a transient phenomenon but there may also be a number of routes to each present-day taxonomic class.

What then are the physical processes which led to the remarkably recent postulated transformation of spirals into S0s? A number of hypotheses have been advanced including dynamical friction in the cluster potential, gas stripping of infalling field galaxies by a dense intracluster medium and 
galaxy-galaxy merging induced perhaps by the hierarchical growth of clusters (see Oemler 1992 for a summary of the pre-HST data in the context of these theories). Numerical simulations of some of these effects have been performed by Mihos (1995) and by Moore et al (1996). Semi-analytical calculations have been performed by Balland et al (1997).

Significant advances are now being made by linking the HST imaging data with spectroscopic diagnostics of recent star formation (Couch et al 1994, Dressler et al 1994, Barger et al 1996, Couch et al 1997). A surprising fraction of the spectrally active systems (strong [O II] emission, deep Balmer absorption lines and blue continua) show signs of morphological disturbances; many are suggestive of merging systems, virtually always involving a disk galaxy (Figure 3). In contrast, the spectrally evolved "postburst" systems (no [O II], deep Balmer absorption lines and red continua) are primarily regular Es or S0s (Figure 3). One puzzle introduced by Barger et al in attempting to define a single cycle of activity (driven by merging or strong dynamical friction in the core) relates to the luminosity distributions of these two classes. If the latter population follows the former, a substantial fading in luminosity would be expected; in fact the $K$-band luminosity distribution of the post-burst population is comparable to that of the active group.

It seems unlikely, given the sample size now available, that the absence of luminous blue precursors of the red post-burst galaxies (Figure 3) is a selection effect arising from the short period when they would be visible. A more sensible explanation is that a large proportion of the luminous postburst systems had their star formation truncated about 1-2 Gyr earlier, i.e. no burst occurred. Those bursting galaxies that are observed presumably fade into systems below the current survey limits $\left(M_{V}>-20+5 \log h\right)$ and thus their fate is not yet clear. The existence of at least two distinct processes contributing to the transformation occurring in the rich cluster environment should not be that surprising.

Abraham et al (1996b) propose that truncated star formation initiated for an infalling population of field galaxies could be an important producer of S0s; in support they observe a strong radial gradient in the mean Balmer line strength over $0.4-3 h^{-1} \mathrm{Mpc}$ in the well-studied cluster Abell 2390. Similar spectral signatures have been seen in the outskirts of the Coma cluster (Caldwell et al 1993) and other nearby groups (Caldwell \& Rose 1997) are interpreted in the context of infalling groups whose members suffer some event after a first passage through the intercluster medium in the core. One major puzzle remains with truncated models which bypass the blue burst phase however, namely that the depth of the observed Balmer absorption lines cannot easily be reproduced (Poggianti \& Barbaro 1996).

To summarise, the above studies have been invaluable in demonstrat- 

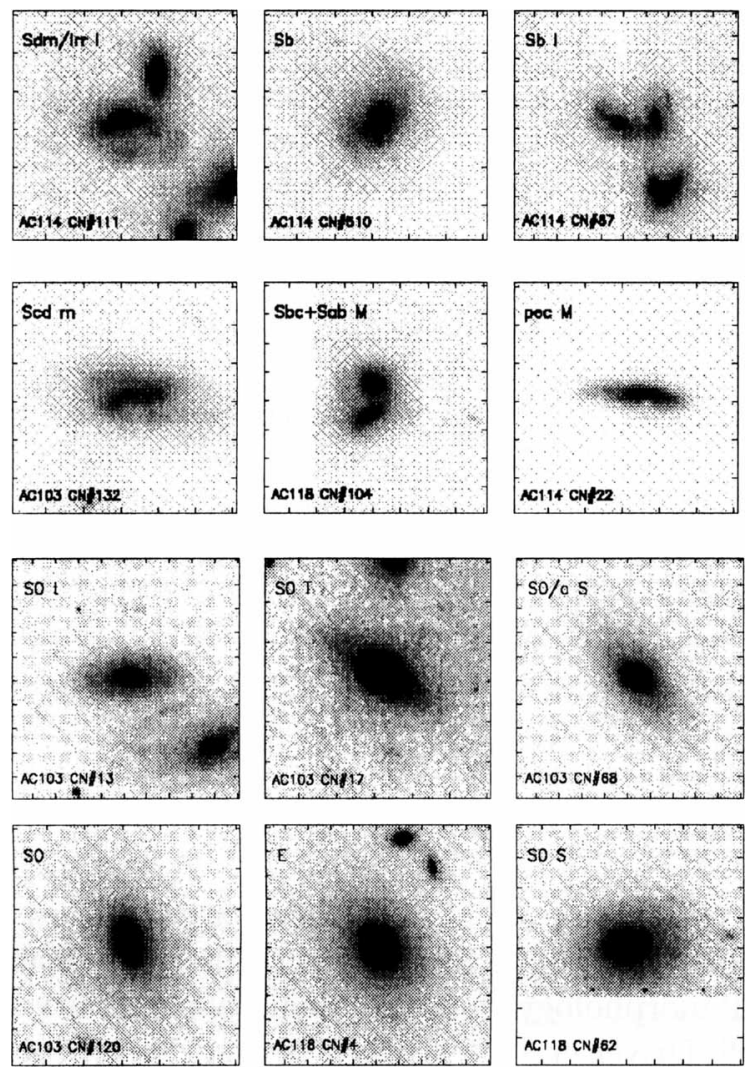

Figure 3. WFPC-2 F814W images of galaxies with intermediate dispersion spectra from the cluster study of Couch et al (1997). Starburst galaxies (top 3) and blue post-starburst galaxies (next top 3) indicate merging is a likely cause for the enhanced activity. Red galaxies with strong $H \delta$ absorption (bottom 6) indicate an advanced stage of decline after recent star formation. Most of these are too luminous to be likely descendents of the starburst galaxies.

ing convincingly that environmental processes do influence galaxy evolution and that the morphology-density relation was produced remarkably recently. The results highlight the transient nature of morphology and consequent uncertainties associated with assuming fixed population densities over a range in look-back time. The combination of spectral diagnostics and HST imaging has been particularly effective. It seems that more than one process may be responsible for the demise of the cluster spirals seen in the HST image. In the cluster core, merging and dynamical friction may excite the abundant gas-rich disks into fresh starbursts and these may fade into lower luminosity spheroidals. However, some S0s, perhaps the most 
luminous examples in the cluster cores, appear to be genuinely old along with the bulk of the ellipticals. A continuous infall of field galaxies may provide an additional source of material for transformation. The overall goal is now to reproduce the luminosity functions of the various morphological types in well-defined samples at various redshifts according to the processes responsible.

\section{The Evolution of Field Galaxies to $z \simeq 1$}

The lessons learnt in the cluster studies concerning the transient nature of a morphological type apply even more so in the lower density 'field' environment where the changes with look-back time are more dramatic (Ellis 1997). Following the theme of synergy between ground and space, there are two results, namely (i) the synthesis of HST imaging with two major redshift samples, the Canada-France (CFRS, Lilly et al 1995) and LDSS (Ellis et al 1996) surveys, and (ii) the integration of these results with deeper work much of which has been inspired by the Hubble Deep Field (HDF, Williams et al 1996).

The CFRS and LDSS redshift surveys reveal significant changes in the luminosity function (LF) have occurred since $z \simeq 1$ that are likely to be luminosity-and colour-dependent. The trends are strongest when attention is confined to the star-forming component (Ellis 1997). Galaxy counts subdivided by HST morphology from the Medium Deep Survey (MDS, Glazebrook et al 1996, Driver et al 1996) likewise indicate dominant trends for a sub-population of galaxies with irregular morphology. Do these results mean the bulk of the recent field evolution is restricted to one sub-luminous class of galaxy?

The availability of HST morphology and spectroscopic redshifts for a large homogeneous sample has been slow to emerge, because of the mismatch in field of view between WFPC-2 and efficient ground-based multiobject spectrographs. By mosaicing WFPC-2 images of ground-based redshift survey fields, the CFRS and LDSS teams have together constructed a sample of over 300 galaxies with $I<22$ and $B<24$ spanning the redshift range $0<z<1.2$. A high proportion of the sample fall into the category of galaxies with irregular morphology, with good agreement between those so defined visually and via the automated measurement of assymetry and concentration. With the benefit of redshifts, the contribution of these sources to the overall redshift trends can, for the first time, be examined (Brinchmann et al 1997).

The new survey indicates a dramatic increase with redshift in the fraction of irregular sources (Figure 4), significantly more than can be accounted for by late-type spirals misclassified as irregulars because of redshift- 

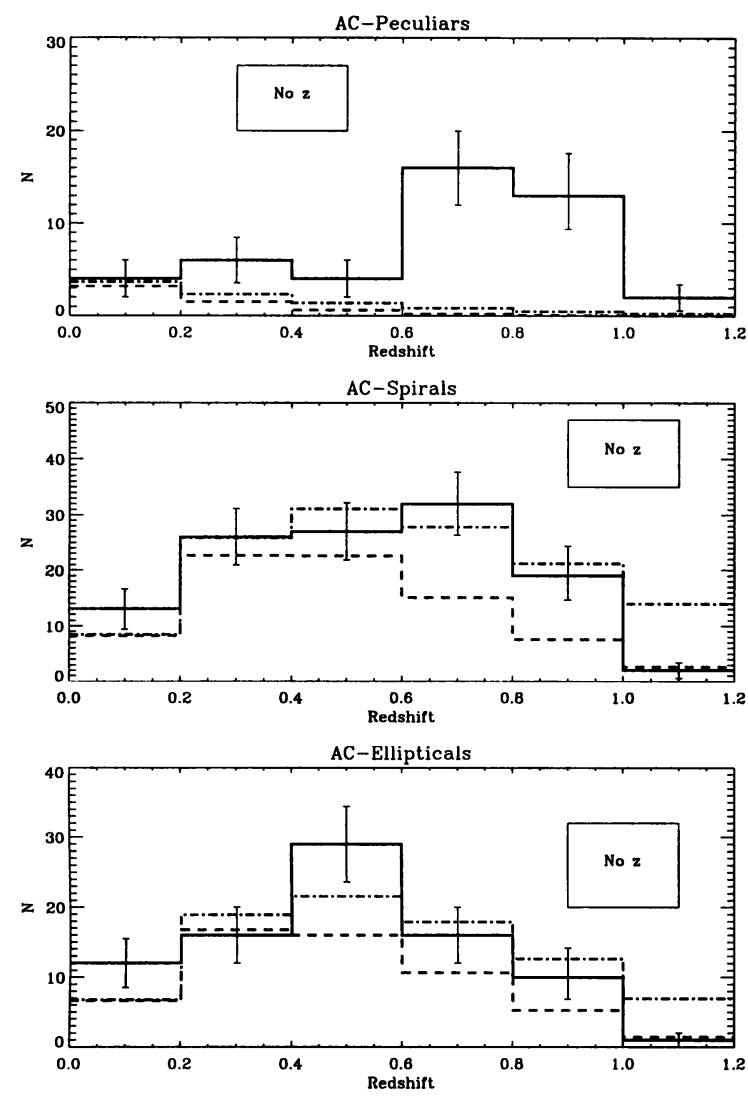

Figure 4. The redshift distribution of galaxies in the CFRS/LDSS HST imaging survey divided according to a morphological criterion based on concentration and asymmetry (after Brinchmann et al 1997). AC-P, AC-S, AC-E refer respectively to irregular/peculiars, spirals \& 9 spirals. Model predictions assume no evolution (dashed) and mild evolution (dash-dotted, corresponding to 1 mag. of luminosity evolution in rest-frame $B_{A B}$ at $z=1$ ).

dependent effects (Brinchmann et al 1997). By contrast only modest luminosity evolution is necessary to account for the redshift distribution of the regular spirals and ellipticals. Most importantly, the comoving volume density at $z \simeq 0.8$ is comparable to local estimates. Most of the rise with redshift in the blue luminosity density appears to originate in the irregular/peculiar population although spirals still dominate the overall flux at $z \simeq 1$ (Figure $5)$.

Do the distant irregulars represent a truly independent population of rapidly-evolving systems or are they normal systems rendered irregular by increased star formation? One indication that the irregulars are not "agitated spirals" is that their marked decline in number as redshift decreases 


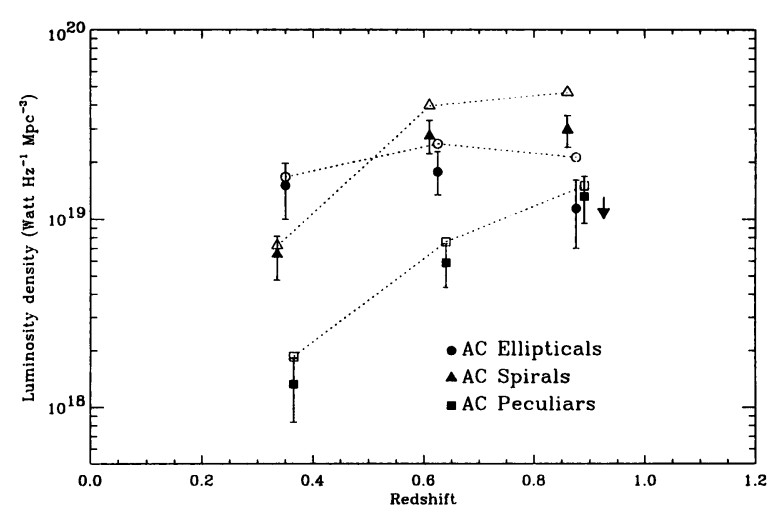

Figure 5. The $B_{A B}$ luminosity density of galaxies detected in the CFRS/LDSS HST imaging survey as a function of redshift and class. The downward arrow indicates the degree to which redshift-dependent effects cause a spurious migration of true spirals into apparent irregular/peculiars. Open symbols refer to estimated integrated values extrapolating the luminosity function below the detection limits (after Brinchmann et al 1997).

is not accompanied by any obvious increase in the number density of regular systems. It turns out this is not a particularly convincing argument, however, since the star forming irregulars would presumably fade as they turned into well-behaved galaxies and many of these may lie below the CFRS/LDSS magnitude limits.

A more convincing measure of the slowly-evolving population of disk galaxies can be derived from structural parameters such as bulge/disk ratios, scale lengths and central surface brightnesses following profile fitting techniques developed by Schade et al (1996). A disk size function, $\Phi\left(\alpha^{-1}\right.$ kpc) $\mathrm{Mpc}^{-3}$, has been constructed by Lilly et al (1997) taking account of flux and surface brightness limits using an analog of the $V / V_{\max }$ test. This size function is only moderately sensitive to the cosmological world model and indicates that large spirals $\left(\alpha^{-1}>2 h^{-1} \mathrm{kpc}\right)$ have more or less the present volume density at $z \simeq 0.8$. Surface photometry suggests only modest luminosity evolution $(<0.5 \mathrm{mag})$ and limited size growth $(<20 \%)$ over the redshift range of the survey $(0.3<z<1)$.

What then is the physical origin of the star forming irregulars which dominate the evolutionary changes seen to $z \simeq 1$ ? Broadhurst et al (1988) proposed, on the basis of spectroscopic arguments similar to those discussed in $\S 2$, that sub-luminous galaxies are being rendered visible by short-lived bursts of star formation. Both infrared luminosities (Cowie et al 1996) and emission line widths (Guzman et al 1996) indicate the rapidly-evolving objects have low masses. Depending on the physical origin of this enhanced star formation and the nature of the underlying stellar population, it is easy 
to see how an irregular system would be produced for a limited period. Alternatively, some appear to be truly compact and similar to extragalactic $\mathrm{H}$ II regions (Guzman et al 1997) whilst others appear to be merging (LeFevre et al 1997).

The Broadhurst et al burst model unfortunately implies the presence of an even greater abundance of low mass systems in a quiescent state which serve as a reservoir for this activity. The outstanding problem is to find examples in the quiescent phase and ultimately the present day faded remnants of this population (amusingly, this is the opposite of the problem we encountered in §2). With a normal initial mass function, it is difficult to exceed a fading of more than 3 magnitudes in rest-frame $B$ since $\mathrm{z} \simeq 0.5$ (Phillipps \& Driver 1995, Babul \& Ferguson 1996) so the absence of a dominant component of red systems with $M_{B} \simeq-16+5 \log h$ remains a puzzle.

We might make progress towards resolving this long-standing riddle along the following lines. Foremost, it is reasonable to support that the rapidly evolving component could be the product of several processes that might befall an abundant population of gas-rich dwarfs expected at early times. The classification of the star forming galaxies outside the range of the regular Hubble sequence has perhaps mistakenly led to a search for a single physical process whereas in fact, one of several mechanisms that leads to an increase in star formation rate can produce these systems. For each mechanism (agitation, fading, disruption, merging, misclassification), a different "remnant" results and meeting the constraints on each would not be so demanding. This is particularly so when it is realised that our knowledge of the present day galaxy population is fairly inadequate at $M_{B}>-16$, especially in absolute volume densities (Ellis 1997).

In summary, the combination of HST and ground-based redshift surveys has shown a surprising contrast between the modest evolution found for the regular disk galaxies and the large increase in number density for irregular systems. Only a small fraction of this irregular population can be explained through mistaken classification problems arising from surface brightness and redshift effects. Whilst it is tempting to search for a single mechanism to explain this dominant activity in the evolution of the luminosity density to $z \simeq 1$, possibly the phenomenon is the manefestation of several distinct processes whose common theme is simply increased star formation (Figure $5)$.

\section{Beyond $z \simeq 1$}

With the systematic study of the $z<1$ population well underway, it is appropriate to now consider the best strategies for surveying the population 
beyond a redshift of 1 . The 'redshift one barrier' was traditionally broken only by locating spectacularly luminous active galaxies (Lilly 1988, Chambers et al 1990, Rowan-Robinson et al 1992) or gravitationally-magnified sources (Ebbels et al 1996, Franx et al 1997). More recently, through Lyman-limit imaging and Keck spectroscopy a population of luminous star forming galaxies has been systematically explored in the redshift window $2.3<z<4.0$ (for a recent review see Pettini et al 1997). This much-heralded observational breakthrough has been accompanied by considerable investigation of the robustness of the Lyman-limit selection criteria (Madau et al 1996), the physical significance of the properties of the galaxies so found (Steidel et al 1996,1997) and the understanding of the results in the context of hierarchical pictures (Baugh et al 1997).

A surprising result from the Lyman-limit galaxy surveys, but one that was, in fact, tentatively suggested from earlier results (Guhathakurta et al 1990 ) is that the inferred star formation rate per unit comoving volume at $z \simeq 3$ is considerably lower than that at $z \simeq 1$. At the current moment, more is known about the nature of the visible galaxy population at $z \simeq 2$ 3 than immediately beyond $z=1$. This curious state of affairs is purely a by-product of the technique used to locate the high $z$ sources which cannot yet be implemented at redshifts below $z \simeq 2.3$. Despite the rapid progress, ultimately we will still need to extend the traditional redshift surveys beyond $z=1$ (see $\S 5$ ).

The rising star-formation density to $z \simeq 1$ and the inferred decline thereafter can be tracked with some uncertainties by inferring redshifts from multiband photometry. In this way, Connolly et al (1997) analyse the HDF dataset to delineate a history of the volume-averaged star formation rate (SFR) whose peak occurs at a surprisingly low redshift. However, in the all-important region $1<z<2$, the reliability of the photometric redshift technique is largely unchecked. Moreover, whereas the validity of the technique is often justified by comparisons with spectroscopic redshifts to $I \simeq 24$ (Lanzetta et al 1997, Hogg et al 1997), in practice the science conclusions are based on an application of the method to much fainter data. A crosscomparison between two independent applications to the HDF dataset to $I<26$ revealed disappointing agreement (Figure 6). Furthermore, a fundamental assumption of the method as commonly used is that the spectral energy distribution of a high redshift galaxy evolves in such a way that it always somehow lies along the locus of present-day equivalents. Given the number of puzzling results discussed for $z<1$ galaxies $(\S 2,3)$ this seems unjustified.

The photometric redshift technique would be useful if it could be proven to yield reliable redshifts at the $\Delta z \simeq \pm 0.3$ level to, say, $I=25$. This is a reasonable goal to verify given the spectroscopic capabilities of $8-10 \mathrm{~m}$ 


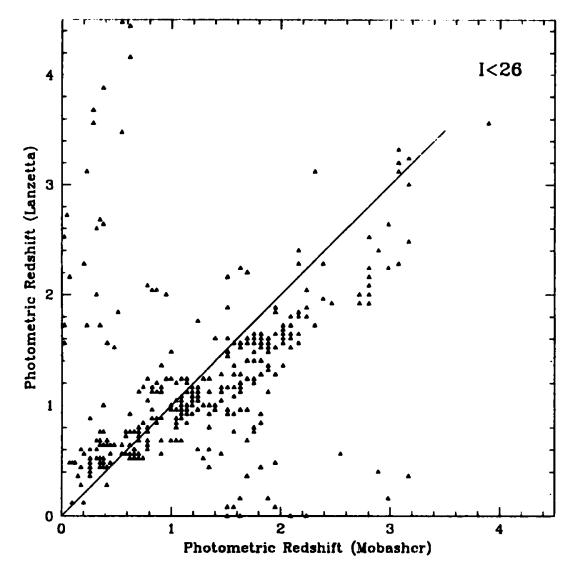

Figure 6. A comparison of the published photometric redshifts from Lanzetta et al (1996) and Mobasher et al (1996) for the Hubble Deep Field to $I=26$, i.e. well beyond the limits of Keck spectroscopy (Ellis 1997).

telescopes. Currently, however, there is little evidence that beyond $z=1$ where useful information is sought. Indeed, there is not yet convincing evidence that the precision of the multi-band fitting method is any better than that indicated by the simple location of the Lyman limit. Nonetheless, even this crude precision is sufficient to support the overall picture of a recent peak of star formation.

In interpreting the star formation histories quantitatively, several caveats apply. Firstly, the SFR is based only on the detected emission from galaxies at optical and uv wavelengths. Dust will not only lead to underestimates of the SFR but also obscured sources will re-radiate at wavelengths where there is currently very little data; this needs to be accounted for in the global calculations (Fall et al 1996, Madau et al 1996). Secondly, different techniques have been applied to infer the SFR in different redshift regimes, and flux limited samples have been corrected to integrated values by making assumptions about the undetected population. Madau (1997) discusses the limitations in detail. With the exception of dust, it is difficult to see how any of these caveats could seriously distort the qualitative picture.

Recent discussion concerning dust has centred on two themes. Firstly, the spectra of the Lyman-limit galaxies show features supportive of high star formation rates yet, puzzlingly, the slopes of their UV continua do not match that expected for young stellar populations (Pettini et al 1997, Calzetti 1997). If this discrepancy is due to dust, it would lead to a fairly modest upward correction in the star formation density. More serious is the objection, from studies at far infrared and sub-mm wavelengths (Mann et 
al 1997, Rowan-Robinson et al 1997, Smail et al 1997c), that even a small number of sources indicative of very high SFRs found at these wavelengths cannot readily be reconciled with the SF history derived from primarily optical data. At the time of writing, the statistics justifying this view are hopelessly inadequate and a key point here is to understand how dramatic modifications to the 'hierarchically approved' SF history could be reconciled with the low metallicity of the Lyman-limit galaxies (Lowenthal et al 1997), the Lyman alpha forest (Songaila \& Cowie 1996) and current limits on the far-IR background (Puget et al 1996).

\section{The Future}

The picture emerging is only empirical and the greatest progress made has been concerned with the integrated properties of the galaxy population. To make progress in understanding the physical basis of the evolutionary trends - for example to test whether the mass assembly rate in stars is consistent with hierarchical theories - we must begin to break the samples into subclasses located by colour, line strength and HST morphology. The above discussion indicates the way each of these observables may be transient and hence not conserved in populations based on normal observational selection. Accordingly, we will need to be much more imaginative in securing and intepreting data that will rise to this challenge.

I suggest there are two logical next steps. The first is to systematically explore the supposed "peak" of SF activity at $1<z<2$ spectroscopically. Paradoxically this region is relatively unexplored notwithstanding the progress made via Lyman limit surveys at higher redshift. The only reliable technique to extend the luminosity function studies begun so successfully at $z<1$ requires $K$-selected spectroscopy with near-infrared instruments sensitive to narrow emission lines. As the IR background is dominated in the $J$ and $H$ windows by $\mathrm{OH}$ emission, its suppression via hardware or software methods is essential. Given the preponderance of blue star-forming systems at these limits, emission line-based surveys should be highly successful (Figure 7).

The second step is to exploit the resolved data on high $z$ sources made available from HST in order to commence linking the trends observed to dynamical data. Line widths (Guzman et al 1996) and rotation curves (Vogt et al 1996) represent an important step forward, but 2-D velocity fields for selected irregular galaxies, such as those in the HDF, should also be attempted using the new generation of integral field spectroscopic units and linked with multicolour analyses (Abraham et al 1997, Figure 7). The challenge of interpreting such data will be considerable but it is now necessary to move the subject from statistical descriptions of an empirical 

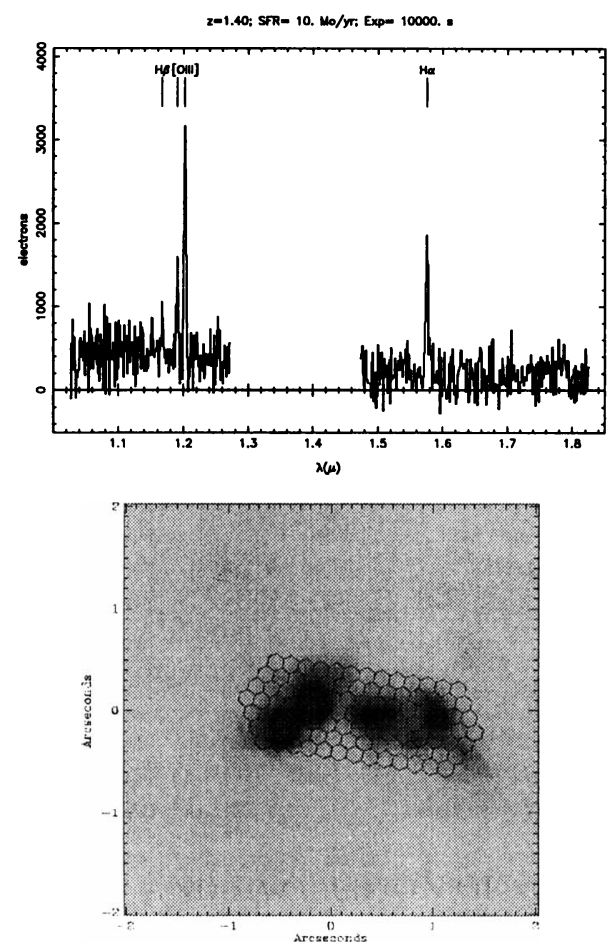

Figure 7. (Top) Simulated infrared spectrum for a star forming galaxy at $z=1.4$ as it would be observed with a 4-m telescope using the Cambridge OH-suppression infrared spectrograph (COHSI, Piché et al 1997). Such instrumentation will allow an extension of well-proven spectroscopic techniques into the important redshift range $1<z<3$. (Bottom) COHSI's integral field unit overlaid on the HST image of a $z=1.355$ HDF galaxy illustrating the possibility of resolved spectroscopic and dynamical data. Each lenslet samples a field of diameter 0.15 arcsec $\left(\simeq 0.6 \mathrm{~h}^{-1} \mathrm{kpc}\right)$.

nature into ones more closely related to galactic structure, dynamics and the astrophysical origin of star formation on galactic scales.

\section{Acknowledgements}

I acknowledge numerous discussions with my colleagues at Cambridge and collaborators on the Morphs, redshift survey, HST and infrared instrumentation programmes. I thank the IAU organisers and colleagues at NAOJ for generous travel funds that enabled me to participate in this symposium. I also thank the Carnegie Observatories in Pasadena for their hospitality and support during a period of sabbatical leave where this article was completed. 


\section{References}

Abraham, R.G., Valdes, F., Yee, H.K.C. \& van den Bergh, S. (1994) Ap J, 432, 75.

Abraham, R.G., Freedman, W. \& Madore, B. (1996a) in HST and the High Redshift Universe, eds. Tanvir, N.R. et al, World Scientific, p57.

Abraham, R.G., Smecker-Hane, T., Hutchings, J.B. et al (1996b) Ap J 471, 694.

Abraham, R..G., Ellis, R.S., Fabian, A.C., Tanvir, N.R. \& Glazebrook, K. (1997) in preparation

Allington-Smith, J.R., Ellis, R.S., Zirbel, E.L. \& Oemler, A. (1993) Ap J 404, 521.

Aragon-Salamanca, A., Ellis, R.S., Couch, W.J. \& Carter, D. (1993) MNRAS, 262, 764.

Babul, A. \& Ferguson, H.C. (1996) Ap J, 458, 100.

Balland, C., Silk, J. \& Schaeffer, R. (1997) Ap J, in press (astro-ph/9711036)

Barger, A., Aragon-Salamanca, A., Ellis, R.S. et al (1996) MNRAS 279, 1.

Baugh, C., Cole, S. \& Frenk, C.S. (1997) Ap.J. in press (astro-ph/970311)

Bender, R., Saglia, R.P. \& Ziegler, B. (1997) in The Early Universe with the VLT, ed. Bergeron, J., Springer, p105.

Bower, R.G., Lucey, J.R., Ellis, R.S. (1992) MNRAS, 254, 601

Brinchmann, J., Abraham, R., Schade, D. et al (1997) Ap J, in press (astro-ph/9712060)

Broadhurst, T.J., Ellis, R.S. \& Shanks, T. (1988) MNRAS 235, 827.

Butcher, H., Oemler, A. (1978) Ap J, 219, 18.

Caldwell, N. \& Rose, J. (1997) A J, 113, 492.

Caldwell, N., Rose, J.A., Sharples, R.M., Ellis, R.S. \& Bower, R.G. (1993) A J, 106, 473.

Calzetti, D. (1997) in The Ultraviolet Universe at Low and High Redshift, in press (astro$\mathrm{ph} / 9706121$ ).

Chambers, K., Miley, G.K. \& van Breugel, W.J. (1990) Ap J 354, 9.

Connolly, A., Szalay, A., Dickinson, M., SubbaRao, M.U. \& Brunner, R.J. (1997) Ap J, 486, 11.

Couch, W.J., Ellis, R.S., Sharples, R.M. \& Smail, I. (1994) Ap J 430, 121.

Cowie, L., Songaila, A., Hu, E. \& Cohen, J.G. (1996) Ap J, 112, 839.

Dressler, A. (1980) Ap J, 236, 351.

Dressler, A., Oemler, A., Butcher, H. \& Gunn, J.E. (1994) Ap J 430, 107

Dressler, A., Oemler, A., Couch, W.J. et al (1997) Ap J, 490, 577.

Driver, S.P., Windhorst, R.A., Ostrander, E.J, et al (1996) Ap J, 449, 23

Ebbels, T., LeBorgne, J-F., Pello, R. et al (1996) MNRAS 281, 75.

Ellis, R.S. (1997) Ann Rev Astron. Astr. 35, 389.

Ellis, R.S. Colless, M., Broadhurst, T.J., Heyl, J.S. \& Glazebrook, K. (1996) MNRAS, 280, 235.

Ellis, R.S., Smail, I., Dressler, A. et al (1997) Ap J, 483, 582.

Fall, S.M., Charlot, S. \& Pei, Y. (1996) Ap J, 464, 43

Franx, M. , Illingworth, G.D., Kelson, D.D., van Dokkum, P.G. \& Tran, K-Y. (1997) Ap $\mathrm{J}$, 486, L75.

Kauffmann, G. (1995) MNRAS, 274, 153.

Glazebrook, K., Ellis, R.S., Santiago, B. \& Griffiths, R. (1996) MNRAS, 275, 19.

Guhathakurta, P., Tyson, A.J. \& Majewski, S. (1990) Ap J, 357, L9.

Guzman, R., Koo, D., Faber, S.M. et al (1996) Ap J, 460, L5.

Guzman, R., Gallego, J., Koo, D., et al (1997) Ap J, 489, 559.

Hogg, D., Cohen, J. \& Blandford, R. (1997) in The Hubble Deep Field, eds. Livio, M. et al, in press (http://www.sns.ias.edu/ hogg/deep/photoz.html)

Kauffmann, G. (1995) MNRAS 274, 153.

Kelson, D., van Dokkum, P., Franx, M., Illingworth, G.D., Fabricant, D. (1997) Ap J, $478,13$.

Lanzetta, K., Fernandez-Soto, A. \& Yahil, A. (1996) Nature, 381, 759.

Lanzetta, K., Fernandez-Soto, A. \& Yahil, A. (1997) in The Hubble Deep Field, eds. Livio, M. et al, in press (astro-ph/9709166)

LeFévre, O., Ellis, R.S., Lilly, S.J. et al (1997) in HST \&s the High Redshift Universe, eds. 
Tanvir, N.R. et al, World Scientific, p81.

Lilly, S.J. (1988) Ap J, 333, 161.

Lilly, S.J., Tresse, L., Hammer, F., Crampton, D. \& LeFevre, O. (1995) Ap J, 455, 108.

Lilly, S.J., Schade, D., Ellis, R.S. et al (1997) Ap J, in press (astro-ph/9712061)

Lowenthal, J., Koo, D.C., Guzman, R. et al (1997) Ap J, 481, 673.

Madau, P. (1997) in The Hubble Deep Field, ed. Livio, M. et al, STScI Symposium Series, in press (astro-ph/9709147)

Madau, P., Ferguson, H., Dickinson, M.E., Giavalisco, M., Steidel, C.C. \& Fruchter, A. (1996) MNRAS 283, 1388.

Mann, R.G., Oliver, S.J., Serjeant, S. et al (1997) MNRAS, 289, 482.

Mihos, C. (1995) Ap J 438, L75.

Mobasher, B., Rowan-Robinson, M., Georgakis, A., Eaton, N. (1996), MNRAS 282, L7.

Moore, B., Katz, N., Lake, G., Dressler, A. \& Oemler, A. (1996) Nature, 379, 613.

Naim, A., Lahav, O., Sodre, L., Storrie-Lombardi, M.C. (1995), MNRAS, 275, 567.

Oemler, A. (1992) in Clusters of Galaxies, ed. Fabian, A., Kluwer, p29.

Piché, F., Parry, I., Ennico, K., Ellis, R.S., Pritchard, J., MacKay, C.D. \& McMahon, R.G. (1997) in Optical Telescopes of Today and Tomorrow, ed. Ardeberg, A., Proc. SPIE, 2871, 1332.

Pettini, M., Steidel, C., Adelberger, K. et al (1997) in Origins, ed. Shull, M. et al, in press (astro-ph/9708117)

Phillipps, S. \& Driver, S.P. (1995) MNRAS 274, 832.

Poggianti, B. \& Barbaro, G. (1996) Astron. Astrophys. 314, 379.

Puget, J-L., Abergel, A., Bernard, J-P, Boulanger, F., Burton, W.B., Desert, F-X. \& Hartmann, D. (1996) Astron. Astrophys. 308, L5.

Rowan-Robinson, M., Broadhurst, T., Oliver, S.J. et al (1992) Nature, 351, 719.

Rowan-Robinson, M., Mann, R.G., Oliver, S.J. et al (1997) MNRAS, 289, 490.

Sandage, A., Visvanathan, N. (1978) Ap J, 225, 742.

Schade, D., Lilly, S.J., LeFevre, O. et al (1996) Ap J, 464, 79.

Smail, I., Dressler, A., Couch, W.J. et al (1997a) Ap J Suppl, 110, 213.

Smail, I., Edge, A.C., Ellis, R.S., Blandford, R.D. (1997b) MNRAS, in press (astro$\mathrm{ph} / 9707231)$.

Smail, I., Ivison, R.J., Blain, A.W. (1997c) Ap J 490, L5.

Songaila, A. \& Cowie, L. (1996) A J, 112, 335.

Stanford, S.A., Eisenhardt, P.R. \& Dickinson, M. (1997) Ap J, in press (astro$\mathrm{ph} / 9708037)$.

Steidel, C., Giavalisco, M., Dickinson, M. \& Adelberger, K.L. (1996) A J, 112, 352.

Steidel, C., Adelberger, K., Dickinson, M., et al (1997) Ap J, in press (astro-ph/9708125) van den Bergh (1990) Ap J, 348, 57.

van Dokkum, P. \& Franx, M. (1996) MNRAS, 281, 985.

Vogt, N., Forbes, D.A., Phillips, A.C. et al (1996) Ap J, 465, L15.

Williams, R. , Blacker, B., Dickinson, M. et al (1996) A J, 112, 1335. 